\title{
miR-485-5p improves the progression of ovarian cancer by targeting SRC in vitro and in vivo
}

\author{
Y. YANG, J. LIU*, X. QIAN, Y. LI, Y. WANG, X. XU \\ Department of Gynecology, Third Affiliated Hospital of Qiqihar Medical University, Qiqihar, Heilongjiang, China \\ *Correspondence: liujie0jieliu@163.com
}

Received September 27, 2019 / Accepted January 7, 2020

\begin{abstract}
miR-485-5p is involved in many tumors, but its role in ovarian cancer has been rarely reported. This paper mainly studied the expression and mechanism of miR-485-5p in ovarian cancer. The expression of miR-485-5p in ovarian cancer was compared with that in adjacent tissues, and the expression of miR-485-5p in various ovarian cancer cell lines was detected by RT-PCR. miR-485-5p mimics and pcDNA plasmid or pcDNA-SRC were transfected into SKOV3 cells. Cell proliferation was detected by CCK-8, cell cycle and apoptosis were detected by flow cytometry, and cell migration and invasion were detected by wound assay and transwell assay. Nude mice were inoculated with SKOV3 cells to detect the effect of miR-485-5p on xenograft. SRC and its downstream proteins were detected by western blot. Our data suggested that the expression of miR-485-5p was low in ovarian cancer tissues and ovarian cancer cell lines. miR-485-5p mimics can inhibit the proliferation, migration, invasion, and the induction of cycle arrest and apoptosis of SKOV3 cells in vivo and in vitro, and pcDNA-SRC can reverse the effect of miR-485-5p mimics on ovarian cancer. Our findings suggest that miR-485-5p could inhibit the progression of ovarian cancer by targeting SRC, which might be a new target for ovarian cancer therapy as the SRC inhibitor.
\end{abstract}

Key words: ovarian cancer, miR-485-5p, SRC proto-oncogene

Ovarian cancer is one of the most common gynecological malignancies, ranking the eighth in women with cancer, and the first in mortality rate among gynecological malignant tumor worldwide [1]. Although the prognosis is favorable in the early stage of the disease, it is difficult to detect it incipiently because of the lack of specific symptoms. When the disease is diagnosed, it is in the late stage. The 5-year survival rate of advanced ovarian cancer is less than 30\% [2]. The later the stage, the larger the infiltration area and the higher the malignant degree of the tumors, the prognosis of the patients is worse [3]. At present, the main treatment for ovarian cancer is cytoreductive surgery combined with platinumbased chemotherapy after surgery [4]. With the rapid development of medicine, treatment methods are also constantly breaking through and developing, such as immunotherapy, peritoneal hyperthermia, and traditional Chinese medicine treatment, but there is no significant effect [5]. Therefore, it is important to explore effective approaches for the treatment or diagnosis of ovarian cancer.

For malignant tumors, the activation of oncogene and the inhibition of anti-oncogene are the fundamental causes of the occurrence of such diseases [6]. miRNA, a non-coding single-stranded RNA composed of 22 nucleotide molecules, inhibits post-transcriptional translation by binding specifically to the 3'untranslated region of corresponding mRNA [7]. Current studies have shown that miRNAs can be a new method for the treatment of malignant tumors by targeting to regulate oncogene and anti-oncogene [8]. Xu et al. found that compared with normal gastric tissues, the expression of miR-146B was lower in gastric cancer tissues, and it could negatively regulate $\mathrm{PTP} 1 \mathrm{~B}$ to inhibit proliferation and promote apoptosis in gastric cancer cells [9]. The expression of miR-485-5p is low in many tumors as an anti-oncogene, such as hepatocellular carcinoma, gastric cancer, bladder cancer, breast cancer, melanoma, and glioma [10-12]. However, the function of miR-485-5p in ovarian cancer has rarely been reported.

SRC is an oncogene discovered in 1976 by Bioshop et al. [13]. As the earliest member of the SRC kinase family, SRC kinase is involved in cell proliferation, migration and invasion, angiogenesis, and intracellular transport [14]. It has been confirmed that SRC is overexpressed and activated in advanced ovarian cancer and human ovarian cancer cell lines, but not in normal ovarian epithelial cells. When SRC activa- 
tion was inhibited, the growth of ovarian cancer cells was inhibited, the invasive ability was reduced, and the tumorigenic ability in nude mice was reduced [15]. Recent studies have found that miRNAs are involved in the regulation of the SRC pathway. miR-34a in breast cancer cell lines inhibited proliferation and invasion and promoted sensitivity to dasatinib by targeting c-SRC [16]. Liao et al. demonstrated that miR-1 could suppress proliferation and promoted apoptosis in esophageal cancer cells by inhibiting Src [17].

The relationship between miR-485-5p as well as SRC and ovarian cancer has rarely been reported. In our study, the expression of miR-485-5p was downregulated, and SRC was upregulated in ovarian cancer. We hypothesize that miR-485-5p could inhibit the proliferation, invasion, and apoptosis of ovarian cancer cells by regulating SRC. Our results confirmed this, and the upregulation of miR-485-5p could weaken the tumorigenicity in nude mice.

\section{Materials and methods}

Tissue samples. Tumor tissue samples were collected from 32 patients with ovarian cancer who underwent surgery in the Third Affiliated Hospital of Qiqihar Medical University from January 2015 to September 2018, and paracancerous tissues were collected as a control group. All patients' medical records are complete, and the collection and use of specimens are informed consent of patients. All methods and experimental protocols were approved by the ethics committee of the Third Affiliated Hospital of Qiqihar Medical University.

Cell culture and transfection. Human ovarian cancer cell lines (SKOV3, HO8910, A2780, CoC1) and immortalized normal ovarian epithelial cells (HOSEpIC) were purchased from the American Type Tissue Culture Collection (Manassas, VA) and incubated in 90\% RPMI1640 containing $10 \%$ fetal bovine serum and $1 \%$ penicillin-streptomycin at $37^{\circ} \mathrm{C}$ in $5 \% \mathrm{CO}_{2}$, water-saturated atmosphere.

miR-485-5p mimics and control oligonucleotides, empty vector, and pcDNA3.1 cloned with SRC were synthesized by GenePharma Company (Shanghai, China). When the density of cells reached $70-80 \%$, cells were cultured in serum-free media for $2 \mathrm{~h}$ and transfected using Lipofectamine 2000 according to the manufacturer's instructions. After recovery in fresh medium for $24 \mathrm{~h}$, subsequent experiments were performed.

Quantitative real time PCR. Total RNA in cells or tissues was extracted using Trizol (Sigma). For miRNAs, the DNA was synthesized using one step PrimeScript RNA Gene Synthesis Kit (TaKaRa), and qPCR was performed using One Step SYBR PrimeScript ${ }^{\mathrm{TM}}$ qPCR Kit (TaKaRa) according to the instructions. U6 was used to normalize. For mRNA, the DNA was synthesized using $\mathrm{RT}^{2}$ First Strand Kit (TaKaRa), and qPCR was performed using $\mathrm{RT}^{2}$ qPCR Prime assay (TaKaRa) according to the instructions. GAPDH was used to normalize. The primers were as follows: miR-485-5p forward 5'-TGGCAAGATCACCAGACGG-3' and reverse 5'-GGCACCTTTCGTGGTCTCAC-3'; U6 forward 5'-CTCGCTTCGGCAGCACACA-3' and reverse 5'-AACGCTTCACGAATTTGGGGGT-3'; SRC forward 5'-CACTCGCTCAGCAGGACAG-3', and reverse 5'-AGAGGGCAGTAGGCAGCCTTTCG-3'; and GAPDH forward 5'-CCCCCTCATT-3' and reverse 5'-CCGCATTGCAG-3'.

Luciferase reporter assay. Cells were inoculated into 24-well plates at the density of $1 \times 10^{6}$ cells per well. miR-485-5p mimics or NC and 20 ng SRC-3'UTR-WT or SRC-3'UTR-MUT plasmids were co-transfected into the cells by Lipofectamine 2000 . Fluorescence activity was detected according to the instructions of the dual luciferase reporter assay kit (Promega). The pRL-TK vector (Promega) was used as an internal control.

CCK-8 assay. Cell proliferation was detected using the MTT assay. The cells were inoculated in 96-well plate overnight at the density of $1 \times 10^{4}$ cells per well. After transfected for $0,24,48$, and $72 \mathrm{~h}, 10 \mu \mathrm{l}$ of CCK-8 reagent were added to each well, and further incubated for $4 \mathrm{~h}$ at $37^{\circ} \mathrm{C}$. The absorbance was measured at $490 \mathrm{~nm}$ wavelength by the universal Microplate Reader EL800 (BIO-TEK instruments, Inc., Vermont, MA, USA).

Cell cycle assay. After transfected for 24 hours, the cells were collected and washed twice with PBS, and then 70\% ethanol was added for a fixation overnight at $4{ }^{\circ} \mathrm{C}$. Following that, the cells were collected and washed once with PBS, then RNase $50 \mu \mathrm{g} / \mathrm{ml}$ was added. Cells were cultured at $37^{\circ} \mathrm{C}$ in a water bath for $30 \mathrm{~min}$ and then ethidium iodide (PI) staining agent staining was added for $30 \mathrm{~min}$ in the dark, and the cell cycle changes were detected by flow cytometry (BD Biosciences). CellQuest Pro software (BD Biosciences) was used to detect the number of cells in each phase of the cell cycle.

Cell apoptosis assay. After transfected for $48 \mathrm{~h}$, the cells were collected using trypsin. According to the instructions of the Annexin V-FITC/PI double-staining kit, $5 \mu$ Annexin $\mathrm{V}$ and $10 \mu \mathrm{PI}$ staining were added, and incubated for 15 minutes. Then, the flow cytometry (BD Biosciences) and CellQuest Pro software (BD Biosciences) were used to detect the number of apoptotic cells.

Wound assay. Cells were inoculated to a 12 -well plate $\left(3 \times 10^{5}\right.$ cells $)$ and cultured to form a confluent monolayer. Cells were moderately scratched with $10 \mu \mathrm{l}$ sterile micropipette and then were replaced with appropriate culture medium to remove cell debris. After transfected for 48 hours, the cells were photographed with an inverted microscope, and the scratch width was calculated with ImageJ software (National Institutes of Health, Bethesda, MD). Three experiments were done in duplicate.

Invasion assay. Cell invasion was detected by Transwell. After $24 \mathrm{~h}$ transfection, the cell suspension was prepared using a serum-free medium and then added to the upper chamber of Transwell, and the RPMI 1640 medium containing $10 \%$ FBS was added to the lower chamber. After $24 \mathrm{~h}$, non-invading cells on the upper surface of the filter were 
removed with cotton swabs, and cells that invaded through the Matrigel onto the lower side of the filter were fixed with methanol for $20 \mathrm{~min}$, and then stained with crystal violet for $15 \mathrm{~min}$. Three random visual fields were taken under a light microscope to calculate the number of invaded cells.

Western blot. Total protein was extracted from cells with RIPA lysis buffer according to the manufacturer's protocol. Protein concentrations were detected using a bicinchoninic acid (BCA) protein assay kit (ASPEN, USA). Equal amounts of protein were separated by $10 \%$ sodium dodecyl sulfate (SDS) polyacrylamide gels and then transferred onto polyvinylidene fluoride (PVDF) membranes, which were blocked with $5 \%$ skim milk for $1.5 \mathrm{~h}$, and then incubated with primary antibodies overnight at $4{ }^{\circ} \mathrm{C}$ : SRC (\#2108), PI3K (\#4257), p-PI3K (\#4228), AKT (\#2920), p-AKT (\#4060), or GAPDH (\#5174) (Cell Signaling Technology, Danvers,

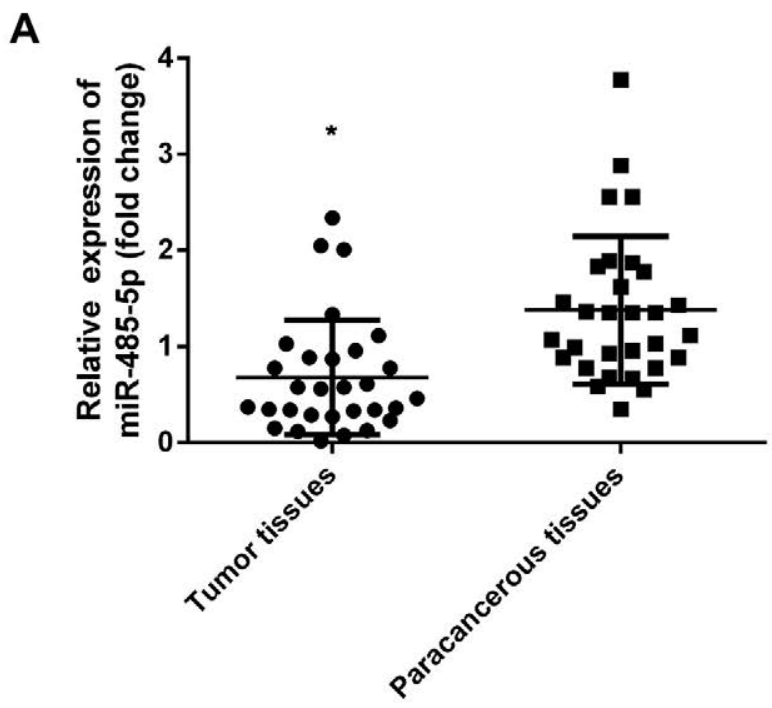

B

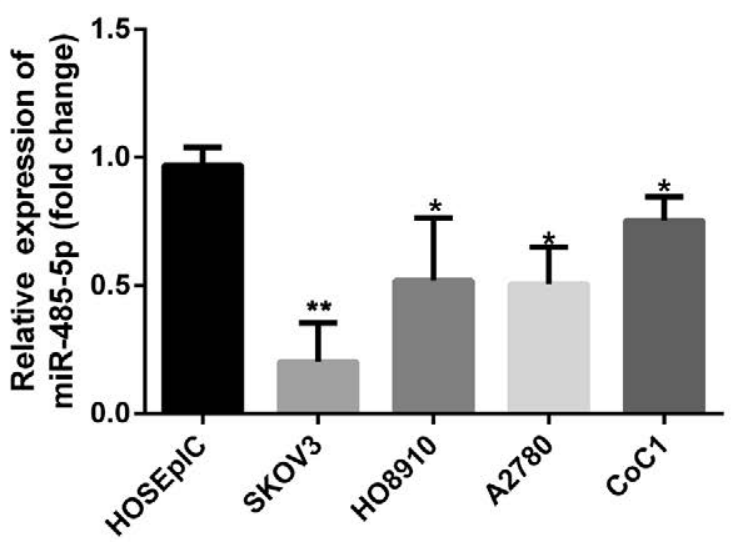

Figure 1. The expression of miR-485-5p was detected in ovarian cancer. A) The expression of miR-485-5p was detected by RT-PCR in ovarian cancer tissues and adjacent tissues. B) The expression of miR-485-5p was detected by RT-PCR in SKOV3, HO8910, A2780, CoC1, and HOSEpIC cells. ${ }^{\star} \mathbf{p}<0.05$ vs. adjacent tissues; ${ }^{\star} p<0.05$ vs. HOSEpIC cells; ${ }^{\star *} p<0.01$ vs. HOSEpIC cells
USA). After washed with TBST three times (10 min each), the membranes were incubated with an anti-rabbit secondary antibody (\#7074, 1:5000) for $1 \mathrm{~h}$ at room temperature. Crossreactivity was visualized using ECL chemiluminescence and then analyzed via scanning densitometry using a Tanon image system. GAPDH was used as a loading control.

Enzyme-linked immunosorbent (ELISA) assay. Total protein was extracted from cells with RIPA lysis buffer according to the manufacturer's protocol. Then, proteins were examined by ELISA for cyclin D1 (hz-1625), caspase-3 (hz-EL-R0160c), and cleaved caspase-3 (E-EL-R0160c) (eBioscience, San Diego, CA) following the manufacturer's instructions.

Tumor growth assay. Female BALB/c nude mice (5-weekold) were obtained from the Shanghai Laboratory Animal Research Center and were used as xenograft ovarian cancer model. The single cell suspension was inoculated subcutaneously into the thighs of nude mice. After inoculation, the nude mice were given free water and diet maintaining aseptic feeding. After visible tumors appeared, 25 nude mice were divided into five groups. A group ( $\mathrm{n}=5)$ : tumors were injected with PBS every other day; B group $(\mathrm{n}=5)$ : tumors were injected with miR-NC every other day; $C$ group $(n=5)$ : tumors were injected with NC agomiR-485-5p every other day; D group $(n=5)$ : tumors were injected with agomiR+pcDNA-plasmid every other day; E group $(n=5)$ : tumors were injected with agomiR-485-5p+pcDNA-SRC every other day. The mice were sacrificed on day 35 after the injection, and the formed tumors were isolated and analyzed, and volume and mass of tumors were measured every 5 days. All animal experiments in this study were performed according to the Health Guide for the Care and Use of the Qiqihar Medical University. The animals were dealt with based on the protocols, approved by the Ethics Committee of the Qiqihar Medical University.

Statistical analysis. GraphPad Prism 5.0 (La Jolla, CA, USA) was used to analyze the results of each experiment. The data are presented in terms of means \pm SE. $t$-test was used for comparison between the two groups, one-way ANOVA was used for comparison among multiple groups, and SNK was used for comparison between two groups. A p-value $<0.05$ was considered to be statistically significant.

\section{Results}

The miR-485-5p level is downregulated in ovarian cancer tissues and cells. To investigate the role of miR-485-5p in ovarian cancer, we detected the expression of miR-485-5p in ovarian cancer tissues and adjacent tissues respectively, and the expression of miR-485-5p in normal ovarian cells and ovarian cancer cell lines was also detected. Our results showed that the expression of miR-485-5p in ovarian cancer tissues was significantly lower than that in adjacent tissues (Figure 1A). Moreover, the expression of miR-485-5p in ovarian cancer cells was lower than that in normal ovarian cells and was the lowest in SKOV3 cells (Figure 1B). 
miR-485-5p directly targets SRC in SKOV3 cells. In recent years, more and more attention has been paid to the role of SRC in ovarian cancer. We predicted that SRC might be one of the targets of miR-485-5p through TargetScan Human 7.2 (www.targetscan.org) (Figure 2A). We detected it by luciferase reporter gene. Our results showed that miR-485-5p mimics could reduce the fluorescence activity of cells transfected with SRC vectors (wt) and had no effect in cells transfected SRC vectors (mut) (Figure 2B). Moreover, WB results showed that miR-485-5p mimics could reduce the expression of SRC in SKOV3 cells. We detected miR-485-5p mimics inhibited the expression phosphorylated PI3K and phosphorylated $\mathrm{AKT}$ and the effects were reversed by pcDNA-SRC (Figure 2C).

A

$$
\begin{array}{rr}
\text { Position 333-340 of SRC 3' UTR } & 5^{\prime} \text {...GAGGGUGCCCUUUUCCAGCCUCA... } \\
\text { hsa-miR-485-5p } & 3^{\prime} \quad \text { CUUAAGUAGUGCCGGUCGGAGA } \\
\text { Mutant position of SRC 3'UTR } & 5^{\prime} \text {...GAGGGUGCCCUUUUCGAGAACCA... }
\end{array}
$$

B

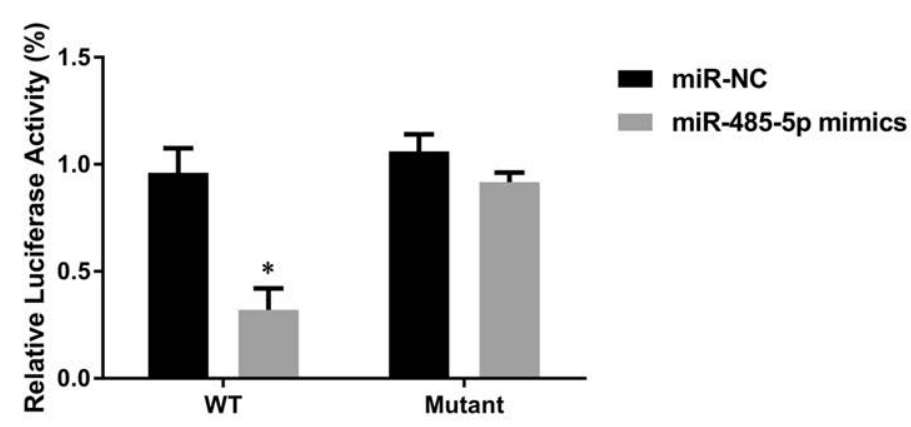

C

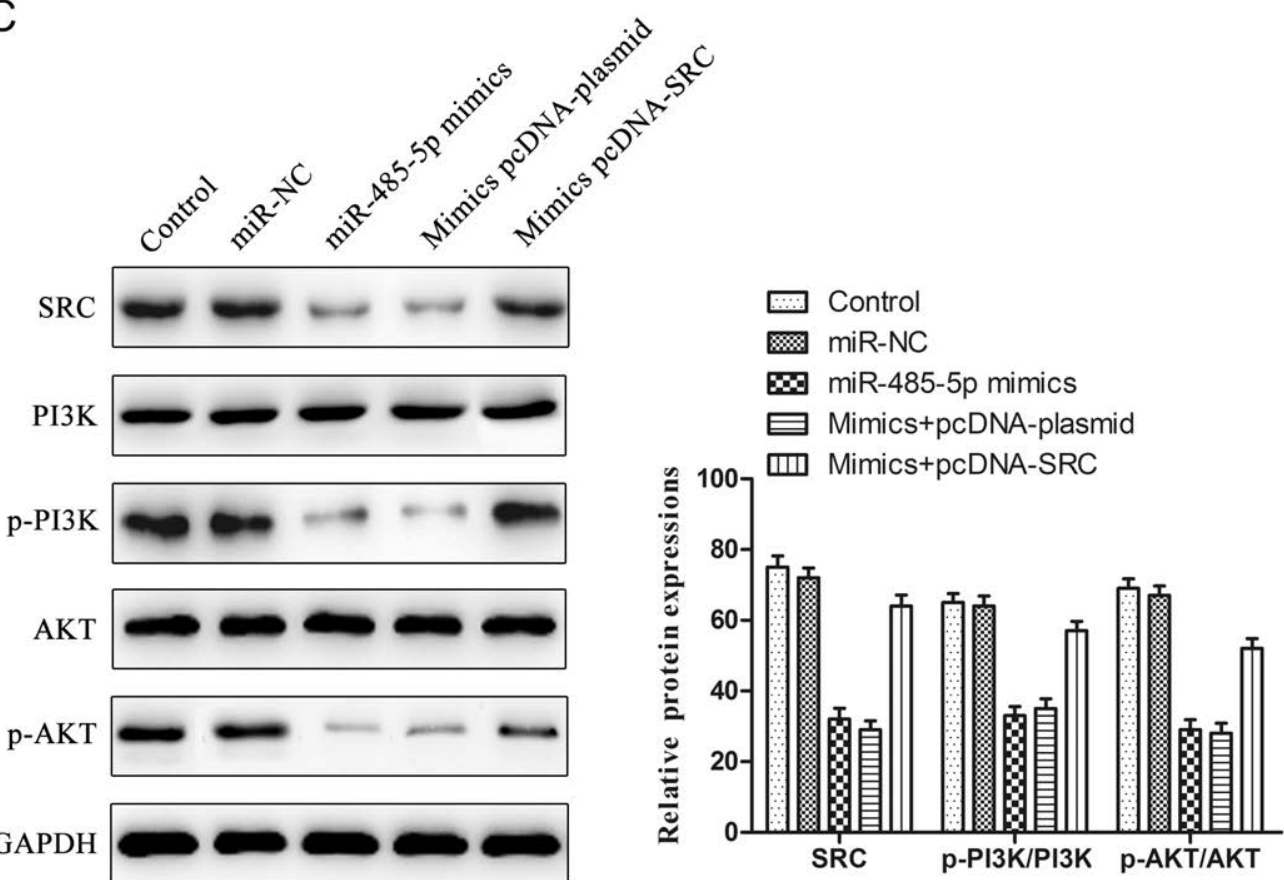

Figure 2. miR-485-5p targeted SRC. A) We predicted that SRC was one of the targets of miR-485-5p through TargetScan Human 7.2. B) We detected miR-485-5p targeted to regulate SRC by luciferase reporter gene assay. C) After cell treatment, we detected the expression of SRC, p-PI3K, and p-AKT by western blot. ${ }^{\star} \mathrm{p}<0.05$ vs. miR-NC; ${ }^{*} \mathrm{p}<0.05$ vs. control; ${ }^{*} \mathrm{p}<0.05$ vs. miR-485-5p mimics 
miR-485-5p inhibits cell proliferation and induces G1 phase arrest and apoptosis in SKOV3 cells by downregulating SRC. The effect of miR-485-5p mimics on SKOV3 cells was measured by CCK-8 assay and flow cytometry assay, in which the role of SRC was detected by transfected pc-DNASRC (Figure 3A). Our data showed that miR-485-5p mimics significantly inhibited cell proliferation compared with control, made G1 phase arrest to $85 \%$ related to $65 \%$ of control (Figure 3B), and induced apoptosis rate from $10 \%$ to $23 \%$ in SKOV3 cells (Figure 3C). However, pc-DNA-SRC reversed those effects, cell cycle (cyclin D1) and apoptosis (cleaved caspase-3) related proteins were detected by ELISA (Figure 3D). The results showed that miR-485-5p could increase the expression of cyclin D1 and cleaved caspase- 3 . The effects of miR-485-5p could be attenuated by pc-DNASRC.

miR-485-5p suppresses cell invasion in SKOV3 cells by downregulating SRC. Wound and transwell assays were used to detected metastatic behaviors of SKOV3 cells. The results showed that miR-485-5p significantly inhibited scratch healing and reduced the number of invasive cells (Figures 4A, 4B). However, overexpression of SRC rescues the effect of miR-485-5p on metastasis of SKOV3 cells. Metastasis (p-FAK, MMP-2, and MMP-9) related proteins were detected by ELISA (Figure 4C). miR-485-5p mimics inhibited the expression of $\mathrm{p}-\mathrm{FAK}, \mathrm{MMP}-2$, and MMP-9, and pc-DNA-SRC reversed the effect.

miR-485-5p inhibits the growth of xenograft tumors by suppressing SRC. The effect of miR-485-5p on tumorigenesis in nude mice was detected by injecting agomiR-485-5p into the appropriate site. Moreover, SKOV3 cells with stable SRC overexpression were established and injected into nude mice to detect the role of SRC. Before the nude mice were sacrificed, the xenograft tumors were photographed (Figure 5A). After the sacrifice, the xenograft tumors were removed and photographed. The results showed that the tumors in the control group were significantly larger than those in the treated group with agomiR-485-5p, and tumors transfected with pcDNASRC blocked the effect of agomiR-485-5p (Figure 5B). In the first two weeks after inoculation, there were no significant differences in the growth of subcutaneous xenograft. After two weeks, the growth of subcutaneous xenograft in control group, control group, miR-NC group and agomiR-485-5p + pc-DNA-SRC group grew rapidly, and the volume and weight of the tumors were larger. However, in the agomiR-485-5p group and the agomiR-485-5p + pc-DNA-plasmid group, the growth of subcutaneous xenograft was slow, the volume of tumors was relatively small, and the weight of tumors was low (Figures 5C, 5D). The expression of SRC was detected by western blot. The results showed that the expression of SRC was decreased by the injection of agomiR-485-5p. In xenograft inoculated with pc-DNA-SRC, the expression of SRC was not reduced by agomiR-485-5p (Figure 5E). So those demonstrated that miR-485-5p suppressed tumor growth by decreasing SRC.
Taken together, miR-485-5p could inhibit cell proliferation, induce cell cycle tissue and apoptosis, as well as inhibit the growth of xenograft by targeting SRC. miR-485-5p may be a treatment target in ovarian cancer as an inhibitor of SRC.

\section{Discussion}

Ovarian cancer is one of the three most common malignant tumors in the female reproductive system, accounting for $50 \%$ to $70 \%$ of primary ovarian tumors. It is the most mortal malignant tumors in female reproductive system tumors, and there are few tumor markers for early diagnosis [3]. miRNAs could participate in the process of cell proliferation, differentiation, and apoptosis and act as a regulator of carcinogenesis or a driving factor of tumor metastasis [18]. In previous work, we found that the expression of miR-485-5p in ovarian cancer tissues was significantly lower than that in adjacent tissues. We calculated that there was a correlation between miR-485-5p and SRC. We confirmed that miR-485-5p can target SRC through luciferase reporter gene detection, and we validated it through western blot. We also found that overexpression of $\mathrm{miR}-485-5 \mathrm{p}$ can inhibit the proliferation, migration, invasion, and induce cycle arrest and apoptosis in SKOV3 cells. Moreover, overexpression of miR-485-5p can inhibit the tumorigenesis of subcutaneous transplantation tumors. We also found that overexpression of SRC could reverse the effect of miR-485-5p on ovarian cancer in vivo and in vitro.

miRNAs are important regulators involved in the process of tumors, which affect their transcriptional expression by targeting specific RNA. miR-485-5p had been reported to be involved in the regulation of multiple tumors. It had been reported that the expression of miR-485-5p was low in osteosarcoma tissues and cells, and the proliferation, invasion, and migration of osteosarcoma cells were inhibited with the upregulation of miR-485-5p induced downregulation of CX3CL1 [19]. The expression of miR-485-5p was low in gastric cancer tissues, and NUDT1 was high in gastric cancer tissues, which might be involved in high metastasis rate and low survival rate in gastric cancer patients. Further studies showed that the low expression of miR-485-5p could promote the proliferation and metastasis of gastric cancer cells by upregulating the expression of NUDT1 [20]. Mitochondrial function and cell migration and invasion of breast cancer cells were inhibited by miR-485-5p and miR-485-3p [21]. Downregulation of miR-485-5p in colon cancer was also reported in vitro and in vivo, and mimetic-induced miR-485-5p upregulation in DLD-1 and SW480 cells could inhibit cell proliferation, migration, invasion, and induce apoptosis by binding to the 3'UTR of CD147 to reduce its expression [22]. However, the expression and mechanism of miR-485-5p in ovarian cancer have rarely been reported. In our article, the results showed that the expression of miR-485-5p was low in ovarian cancer tissues but high in paracancerous tissues. Moreover, the overexpression of miR-485-5p could inhibit the prolif- 

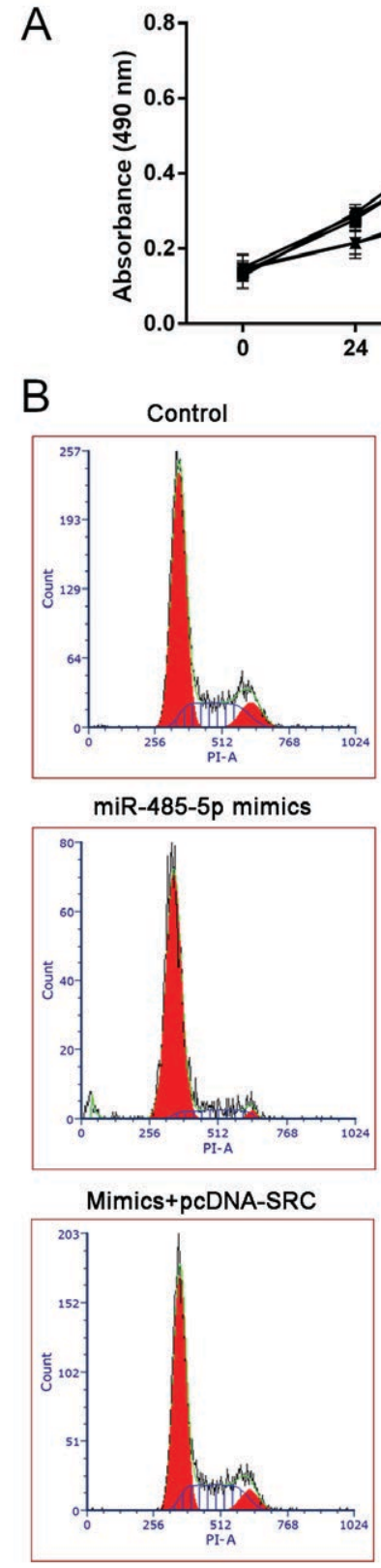

D

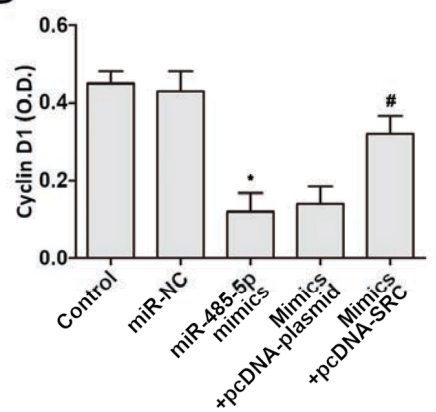

C
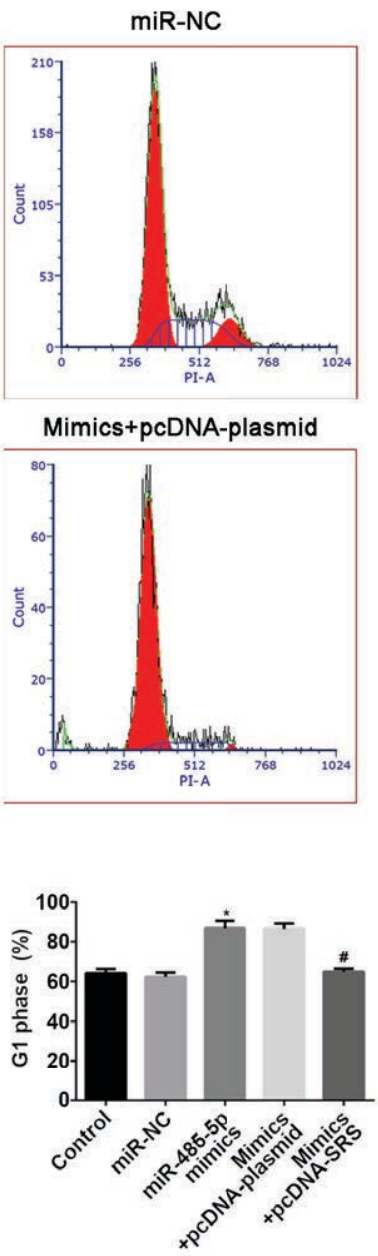
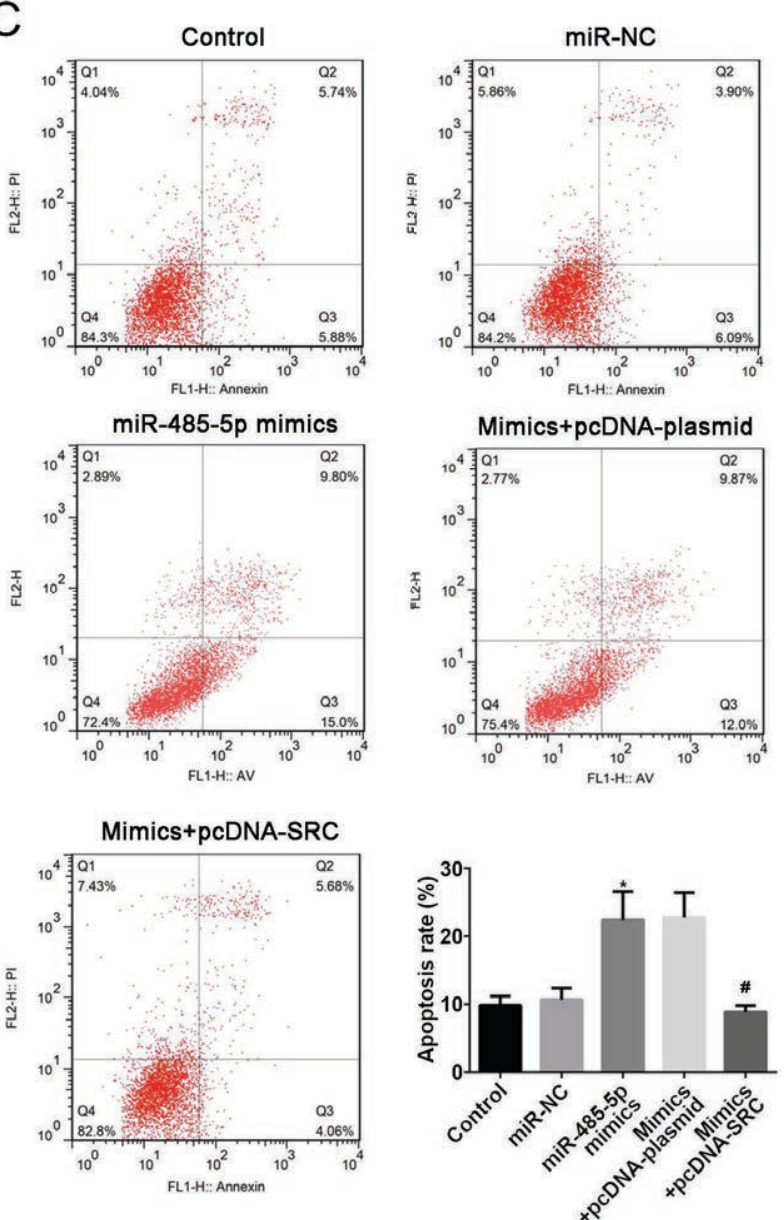

Figure 3. miR-485-5p inhibited SKOV3 cells proliferation, and induced cycle arrest and apoptosis by targeting SRC. A) The proliferation of SKOV3 cells was detected by CCK-8. B, C) Cell cycle and apoptosis of SKOV3 cells were detected by flow cytometry. D) The expressions of cyclin D1, caspase 3 , and cleaved caspase 3 were detected by ELISA. ${ }^{\star} \mathrm{p}<0.05$ vs. control; ${ }^{*} \mathrm{p}<0.05$ vs. miR-485-5p mimics 

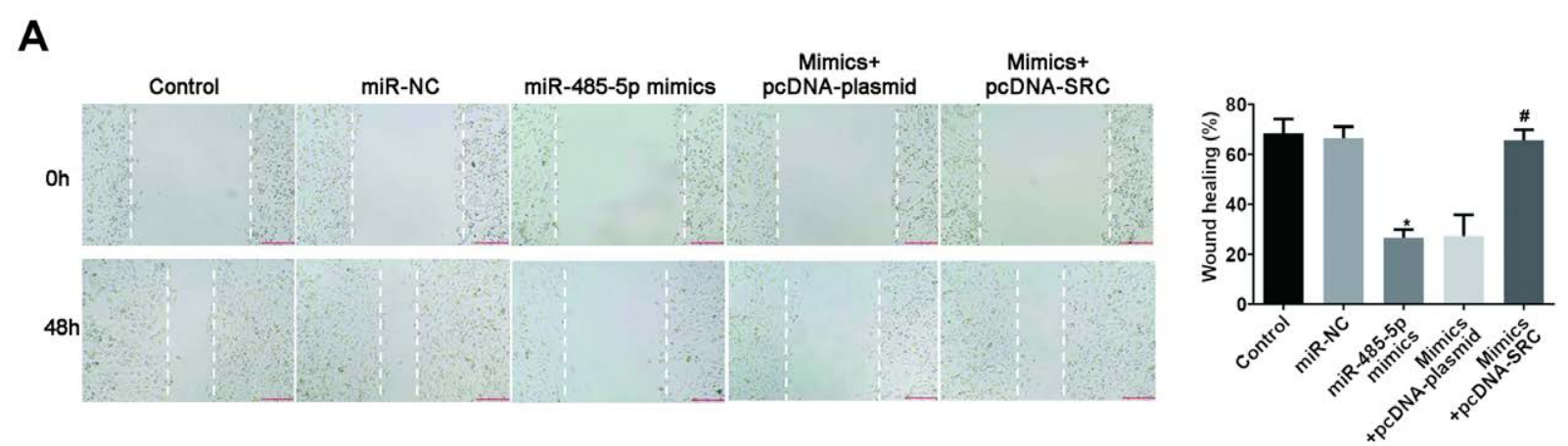

B

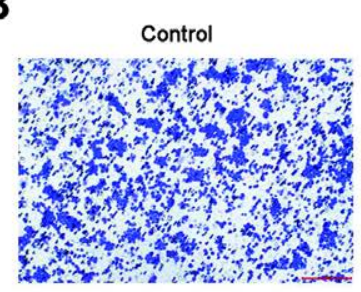

miR-485-5p mimics
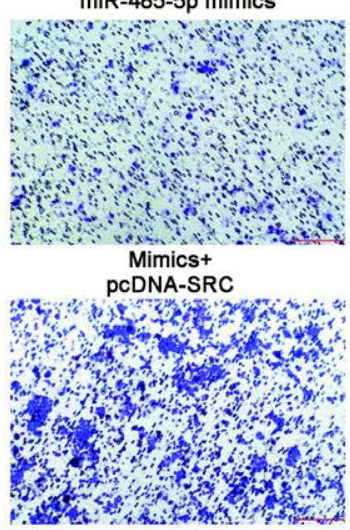

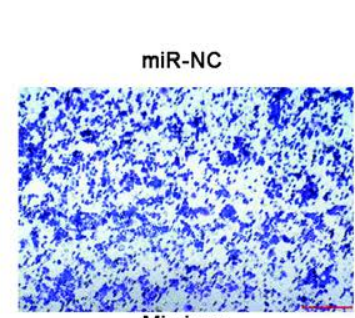

C
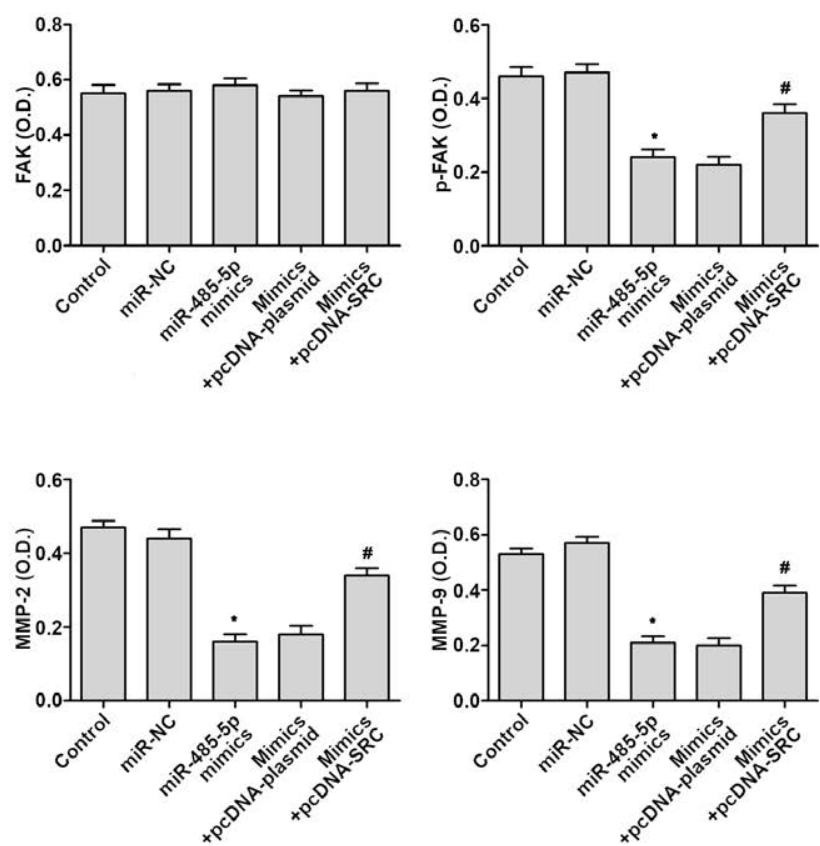

Figure 4. miR-485-5p suppressed SKOV3 cells metastasis by targeting SRC. A) The migration of SKOV3 cells was detected by wound assay. B) The invasion of SKOV 3 cells was detected by Transwell. C) The expressions of FAK, p-FAK, MMP-2, and MMP-9 were detected by western blot. ${ }^{\star}$ p $<0.05$ vs. control; ${ }^{\#} \mathrm{p}<0.05$ vs. $\mathrm{miR}-485-5 \mathrm{p}$ mimics

eration, migration, invasion, and induce cell cycle arrest and apoptosis in ovarian cancer cells. Moreover, the expression of p-PI3K, p-AKT, cyclin D1, p-FAK, MMP-2, and MMP-9 was inhibited by miR-485-5p mimics, and cleaved caspase 3 were promoted. Further studies have shown that the high expression of miR-485-5p can inhibit the growth of ovarian cancer subcutaneous xenograft.

SRC protein is the first member of the discovered SRC family kinases (SFKs). Overexpression and abnormal activation of SRC exist in many malignant tumors. SRC can activate multiple signaling pathways, including the MAPK pathway, FAK pathway, PI3K pathway, etc., which are closely related to a series of malignant biological behaviors, such as tumor cell proliferation, cell-cell junction destruction, tumor invasion, migration, and angiogenesis [23-25]. It had been found that the overexpression of miR-144-3p and the low expression of SRC could significantly inhibit the metastasis of A549 cells induced by TGF- $\beta 1$, and their data also indicated that miR-144-3p participated in the targeting regulation of SRC and regulated the expression and activation of AKT and ERK [26]. miR-34a could inhibit the proliferation and metastasis of TNBC cell lines, and increase the drug resistance of cells to dasatinib by targeting SRC [16]. Overexpression of SRC was detected in human ovarian cancer cells and studies have shown that SRC may be a biomarker or therapeutic target of ovarian cancer $[27,28]$. Whether miR-485-5p can target SRC 
A

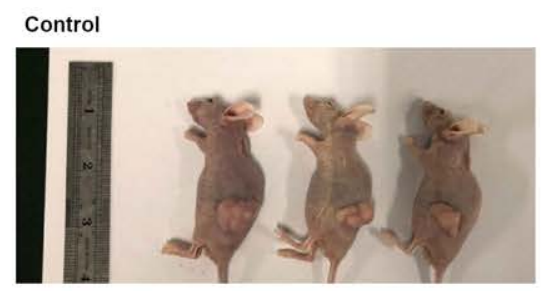

miR-NC

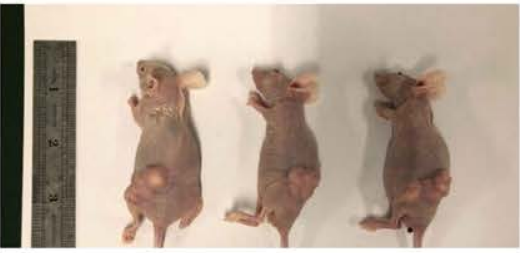

agomiR-485-5p

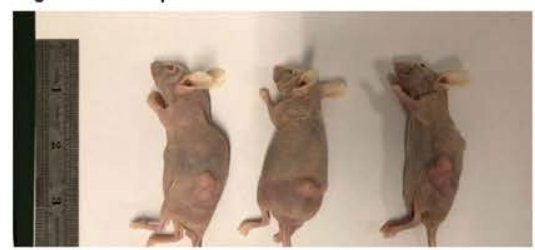

agomiR+pcDNA-plasmid

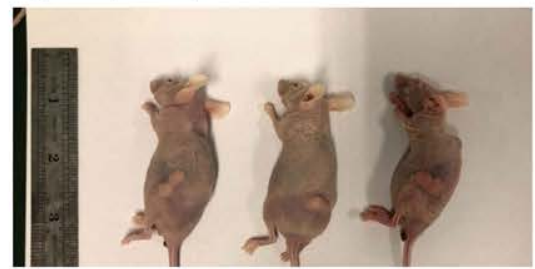

agomiR+pcDNA-SRC

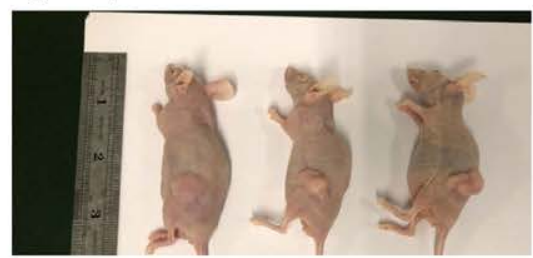

$\mathbf{E}$

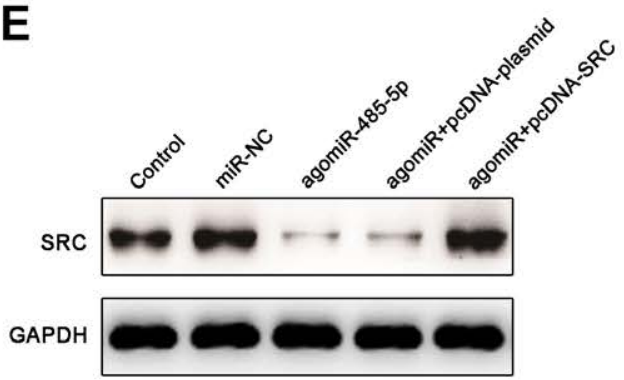

B

C

D
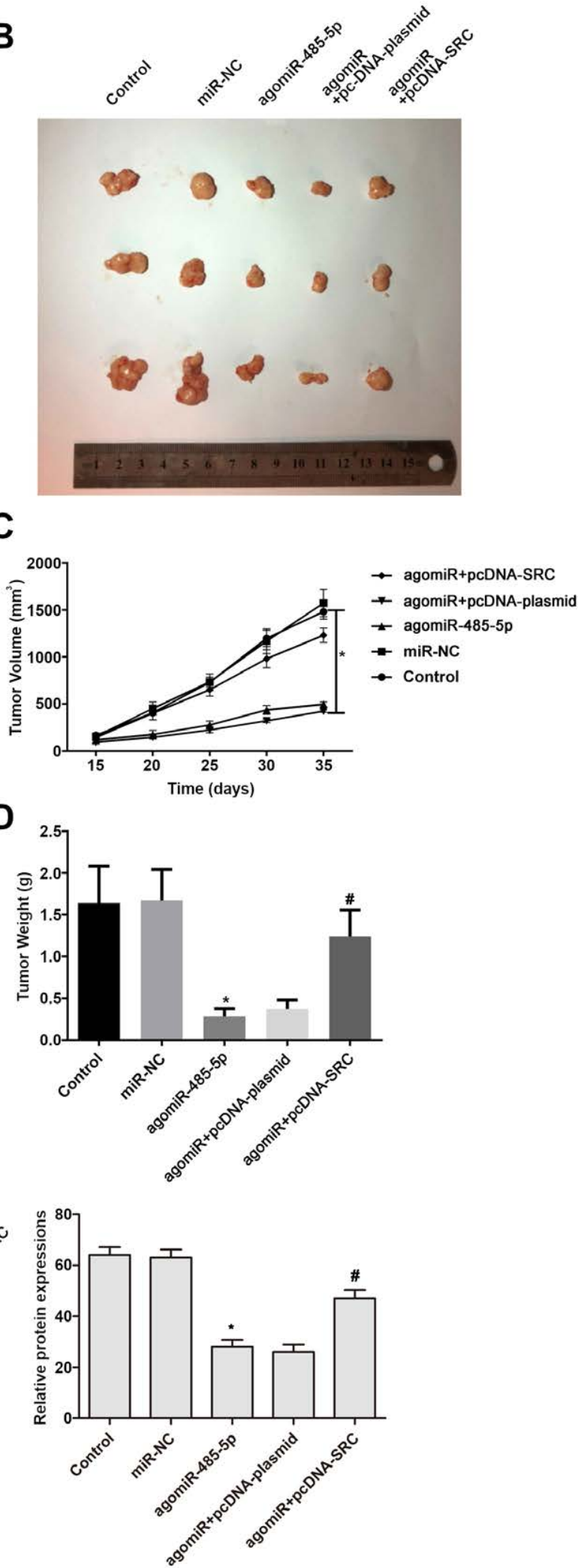

Figure 5. miR-485-5p inhibited the growth of xenograft tumors by targeting SRC. A) Before the nude mice were sacrificed, the xenograft tumors were photographed. B) Separated xenograft tumors were photographed. C) Tumor volume was detected every week. D) Tumor weight was detected every week. E) The expression of SRC was detected by western blot. ${ }^{\star} p<0.05$ vs. control; ${ }^{*} p<0.05$ vs. miR- $485-5 p$ mimics 
in ovarian cancer is uncertain. The results of the luciferase reporter gene showed that the expression of miR-485-5p was downregulated by targeting the 3'UTR of SRC. SRC can affect cell proliferation, cycle, and apoptosis by activating PI3K/AKT signaling pathway [29-31]. Some studies have shown that the Src/PI3K pathway can activate FAK, and this may also occur through the $\mathrm{H} 2$ region of Src linked to the C-terminal tyrosine residues of FAK, thus regulating the expression of MMPs to affect the metastasis of cancer cells $[31,32]$. Our data suggest that pcDNA-SRC can reverse the effects of miR-485-5p on cell proliferation, cycle, apoptosis, and metastasis in vivo and in vitro.

In conclusion, mir-485-5p can inhibit the proliferation, cycle, apoptosis, and metastasis of ovarian cancer cells by downregulating SRC in vivo and in vitro. The results of this study can provide new therapeutic targets for ovarian cancer patients, and provide an experimental reference for the role and mechanism of microRNAs in ovarian cancer.

Acknowledgments: The present study was supported by the Science and Technology Planning Project of Qiqihar, Heilongjiang Province, China (Grant No. SFGG-201962).

\section{References}

[1] JEMAL A, BRAY F, CENTER MM, FERLAY J, WARD E et al. CA Cancer J Clin 2011; 61: 69-90. https://doi.org/10.3322/ caac. 20107

[2] ALETTI GD, GALLENBERG MM, CLIBY WA, JATOI A, HARTMANN LC. Current Management Strategies for Ovarian Cancer. Mayo Clin Proc 2007; 82: 751-770. https:// doi.org/10.4065/82.6.751

[3] HOLSCHNEIDER CH, BEREK JS. Ovarian cancer: Epidemiology, biology, and prognostic factors. Semin Surg Oncol 2000; 19: 3-10. https://doi.org/10.1002/10982388(200007/08)19:1<3::aid-ssu2>3.0.co;2-s

[4] DUFFAUD F, THERASSE P. [New guidelines to evaluate the response to treatment in solid tumors]. Bull Cancer 2000; 87: 881-886.

[5] ZHANG S, BALCH C, CHAN MW, LAI HC, MATEI D et al.: Identificationand characterization ofovariancancer-initiating cells from primary human tumors. Cancer Res 2008; 68: 43114320. https://doi.org/10.1158/0008-5472.CAN-08-0364

[6] CHU P, XU L, SU H. HULC functions as an oncogene in ovarian carcinoma cells by negatively modulating miR125a-3p. J Physiol Biochem 2019; 75: 163-171. https://doi. org/10.1007/s13105-019-00669-5

[7] KARRES JS, HILGERS V, CARRERA I, TREISMAN J, COHEN SM. The conserved microRNA miR-8 tunes atrophin levels to prevent neurodegeneration in Drosophila. Cell 2007; 131: 136-145. https://doi.org/10.1016/j.cell.2007.09.020

[8] FANG T, LV H, LV G, LI T, WANG C et al. Tumor-derived exosomal miR-1247-3p induces cancer-associated fibroblast activation to foster lung metastasis of liver cancer. Nat Commun 2018; 9: 191. https://doi.org/10.1038/s41467-01702583-0
[9] XU J, ZHANG Z, CHEN Q, YANG L, YIN J. miR-146b Regulates Cell Proliferation and Apoptosis in Gastric Cancer by Targeting PTP1B. Dig Dis Sci 2020; 65: 457-463. https://doi. org/10.1007/s10620-019-05771-8

[10] KANG M, REN MP, ZHAO L, LI CP, DENG MM. miR-485$5 \mathrm{p}$ acts as a negative regulator in gastric cancer progression by targeting flotillin-1. Am J Transl Res 2015; 7: 2212-2222.

[11] SUN X, LIU Y, MING L, WANG M, WANG Y. Involvement of miR-485-5p in hepatocellular carcinoma progression targeting EMMPRIN. Biomed Pharmacother 2015; 72: 58-65. https://doi.org/10.1016/j.biopha.2015.04.008

[12] WU J, LI J, REN J, ZHANG D. MicroRNA-485-5p represses melanoma cell invasion and proliferation by suppressing Frizzled7. Biomed Pharmacother 2017; 90: 303-310. https:// doi.org/10.1016/j.biopha.2017.03.064

[13] ROUX ALL, BUSQUETS MA, SAGUÉS F, PONS M. Kinetics characterization of $\mathrm{c}$-Src binding to lipid membranes: Switching from labile to persistent binding. Colloids Surf B Biointerfaces 2016; 138: 17-25. https://doi.org/10.1016/j.colsurfb.2015.11.013

[14] KIM LC, LANXI S, HAURA EB. Src kinases as therapeutic targets for cancer. Nat Rev Clin Oncol 2009; 6: 587-595. https://doi.org/10.1038/nrclinonc.2009.129

[15] HAN LY, LANDEN CN, TREVINO JG, HALDER J, LIN YG et al. Antiangiogenic and antitumor effects of SRC inhibition in ovarian carcinoma. Cancer Res 2006; 66: 8633-8639. https://doi.org/10.1158/0008-5472.CAN-06-1410

[16] ADAMS BD, WALI VB, CHENG CJ, INUKAI S, BOOTH CJ et al. miR-34a Silences c-SRC to Attenuate Tumor Growth in Triple Negative Breast Cancer. Cancer Res 2016; 76: 927939. https://doi.org/10.1158/0008-5472.CAN-15-2321

[17] LIAO Z, WANG X, LIANG H, YU A, REHMAN UU et al. miR-1 suppresses the proliferation and promotes the apoptosis of esophageal carcinoma cells by targeting Src. Cancer Med 2017; 6: 2957-2965. https://doi.org/10.1002/cam4.1214

[18] EICHELMANN AK, MATUSZCAK C, HUMMEL R, HAIER J. Role of miRNAs in cell signaling of cancer associated fibroblasts. Int J Biochem Cell Biol 2018; 101: 94-102. https:// doi.org/10.1016/j.biocel.2018.05.015

[19] WANG FR, XU SH, WANG BM, WANG F. MiR-485-5p inhibits metastasis and proliferation of osteosarcoma by targeting CX3CL1. Eur Rev Med Pharmacol Sci 2018; 22: 7197-7204. https://doi.org/10.26355/eurrev_201811_16253

[20] DUAN J, ZHANG H, LI S, WANG X, YANG H et al. The role of miR-485-5p/NUDT1 axis in gastric cancer. Cancer Cell Int 2017; 17: 92. https://doi.org/10.1186/s12935-017-0462-2

[21] LOU C, XIAO M, CHENG S, LU X, JIA S et al. MiR-485-3p and miR-485-5p suppress breast cancer cell metastasis by inhibiting PGC-1a expression. Cell Death Dis 2016; 7: e2159. https://doi.org/10.1038/cddis.2016.27

[22] HU XX, XU XN, HE BS, SUN HL, XU T et al. microRNA485-5p Functions as a Tumor Suppressor in Colorectal Cancer Cells by Targeting CD147. J Cancer 2018; 9: 2603-2611. https://doi.org/10.7150/jca.24918

[23] SUMMY JM, GALLICK GE. Treatment for advanced tumors: SRC reclaims center stage. Clin Cancer Res 2006; 12: 1398-1401. https://doi.org/10.1158/1078-0432.CCR-052692 
[24] WHEELER DL, IIDA M, DUNN EF. The Role of Src in Solid Tumors. Oncologist 2009; 14: 667-678. https://doi. org/10.1634/theoncologist.2009-0009

[25] AHLUWALIA MS, GROOT JD, LIU W, GLADSON CL. Targeting SRC in glioblastoma tumors and brain metastases: Rationale and preclinical studies. Cancer Lett 2010; 298: 139-149. https://doi.org/10.1016/j.canlet.2010.08.014

[26] JIANG W, XU Z, YU L, CHE J, ZHANG J et al. MicroRNA144-3p suppressed TGF-beta1-induced lung cancer cell invasion and adhesion by regulating the Src-Akt-Erk pathway. Cell Biol Int 2019. https://doi.org/10.1002/cbin.11158

[27] WANG X, JI X, CHEN J, YAN D, ZHANG Z et al. SOX2 Enhances the Migration and Invasion of Ovarian Cancer Cells via Src Kinase. PLoS One 2014; 9: e99594. https://doi. org/10.1371/journal.pone.0099594

[28] ARMAIZ-PENA GN, MANGALA LS, HAN LY, LIN Y, SCHMANDT R et al. Chronic stress promotes tumor growth through a Src-dependent mechanism in a mouse model of ovarian cancer. Brain Behavior and Immunity 2008; 22: 9-10. https://doi.org/10.1016/j.bbi.2008.04.035
[29] CHEN J. The Src/PI3K/Akt signal pathway may play a key role in decreased drug efficacy in obesity-associated cancer. J Cell Biochem 2010; 110: 279-280. https://doi.org/10.1002/ jcb.22572

[30] CHEN B, XU X, LUO J, WANG H, ZHOU S. Rapamycin Enhances the Anti-Cancer Effect of Dasatinib by Suppressing Src/PI3K/mTOR Pathway in NSCLC Cells. PLoS One 2015; 10: e0129663. https://doi.org/10.1371/journal.pone.0129663

[31] XING J, ZHANG Z, MAO H, SCHNELLMANN RG, ZHUANG S. Src regulates cell cycle protein expression and renal epithelial cell proliferation via PI3K/Akt signaling-dependent and -independent mechanisms. Am J Physiol Renal Physiol 2008; 295: F145-152. https://doi.org/10.1152/ajprenal.00092.2008

[32] WILSON C, NICHOLES K, BUSTOS D, LIN E, SONG Q et al. Overcoming EMT-associated resistance to anti-cancer drugs via Src/FAK pathway inhibition. Oncotarget 2014; 5: 7328-7341. https://doi.org/10.18632/oncotarget.2397 